\title{
A rare cause of pleural effusion: adult onset Still's disease
}

\author{
Soner Demirbas, ${ }^{1}$ Orkide Kutlu, ${ }^{2}$ Bahar Kandemir, ${ }^{3}$ Abdullah Sakin ${ }^{2}$ \\ ${ }^{1}$ Department of Chest Diseases, Necmettin Erbakan University Meram Faculty of Medicine, Konya, Turkey \\ ${ }^{2}$ Department of Internal Medicine, Okmeydani Training and Research Hospital, Istanbul, Turkey \\ ${ }^{3}$ Department of Infectious Diseases, Necmettin Erbakan University Meram Faculty of Medicine, Konya, Turkey
}

\begin{abstract}
Adult onset Still's disease is a rare systemic inflammatory disorder. At the onset of the disease sore throat, pharyngitis; which does not respond to antibiotics, one or two times peaking febrile episodes, marked salmoncolored rash on the trunk and extremities, arthralgia, arthritis, myalgia, fatigue, loss of appetite with nausea and weight loss; hepatosplenomegaly and lymphadenopathy can be seen. Among laboratory examinations levels of ferritin and other acute phase reactants distinctly rise, and neutrophilic leukocytosis; ANA and RF negativity are detected. Pleural and pericardial effusions, transient pulmonary infiltration, and rarely myocarditis can be seen during the course of the disease. Here we report a patient who was examined for fever of unknown origin and diagnosed with adult onset Still's disease which is a rare etiology of pleural effusion.
\end{abstract}

Keywords: Adult onset Still's disease; ferritin; pleural effusion.

\begin{abstract}
A dult Onset Still's Disease (AOSD) is a - rarely seen systemic inflammatory disease characterized by high fever, typically marked salmon-colored rash, and arthritis with incompletely known etiopathogenesis. Its incidence has been reported as 1.6 per million individuals. It shows a bimodal age distribution and peaks between $15-25$, and 36-46 years of age, however its incidence is somewhat higher among women. Viral agents as Rubella, Echovirus 7, Epstein Barr virus (EBV), Cytomegalovirus (CMV) and Parvovirus B 19, bacterial infectious pathogenetic factors as Yersinia enterocolitica, and $M y$ -
\end{abstract}

coplasma pneumoniae were held responsible, and its association with genetic factors as HLA B17, B18, B35, and DR2 was reported [1]. With onset of the disease, signs of viral infection and sore throat seen. However non-suppurative pharyngitis is refractory to antibiotics. Febrile episodes follow a "quotidian" pattern, and peak one or two times a day. Body temperature rises above $39^{\circ} \mathrm{C}$, and returns to normal levels at least once a day [2]. Arthralgia, myalgia, fatigue, loss of appetite, nausea, and weight loss can be seen. Signs of high fever, myalgia, rashes, and serositis can demonstrate a diurnal increase. Arthritis demonstrates

Received: July 16, 2014 Accepted: December 11, 2014 Online: September 25, 2015

Correspondence: Dr. Orkide KUTLU. Okmeydani Egitim ve Arastirma Hastanesi, Ic Hastaliklari Klinigi, Istanbul, Turkey. 
a symmetrical mono/oligo or polyartricular onset. At the onset migratory arthritis may gain a permanent, and symmetrical characteristic on the face, and anchylosis may dervelop as a result of destructive processes. Classical salmon-colored transient rash is marked on the trunk, and extensor surface of the extremities, and it can sometimes appear on the face. Rashes become manifest as macular/maculopapular eruptions are generally associated with increasing fever at night. During the course of the disease, hepato splenomegaly, and lymphadenopathies can be seen [3]. Acute phase response is manifested by significant laboratory abnormalities of AOSD including especially marked increase in ferritin, leukocytosis/neutrophilia with ANA, and RF negativity. Though macrophage activation syndrome (MAS) or reactive hemophagocytic syndrome is seen in a small group of people, it is an important complication which requires bone marrow analysis [4].

In our paper, we presented our patient who was hospitalized because of fever of unknown etiology, and diagnosed by us as AOSD. As a rarely seen outcome of AOSD she developed pleural effusion during her clinical follow-up which rapidly responded to steroid therapy.

\section{CASE REPORT}

A-38-year-old female patient consulted to our emergency outpatient clinic with complaints of fever, sore throat, coughing, shivering, chills, joint, and muscle pain, headache, chest pain continuing for nearly 2 weeks and she was hospitalized to investigate the etiology of fever. Physical examination findings were as follows: BP $130 / 80 \mathrm{~mm} \mathrm{Hg}$, body temperature, $39.8 \mathrm{C}^{0}$; HR $120 / \mathrm{min}$; moderately well general physical status with full cooperation, markedly hyperemic pharynx, and tonsils, herpetic rashes around her lips, bilaterally diminished respiratory sounds heard over basal segments and tachycardia. Her neck rigidity was evaluated however the Kernig, and Brudzinsky signs could not be elicited. Laboratory test results were as follows: $\mathrm{Hb} 11 \mathrm{~g} / \mathrm{dl}$ (12.1-17.2), MCV 80.8 fL (82.2-99), WBC 22.10 $\mathrm{K} / \mathrm{uL}$ (4-10), Neu 90.5\% (37-73\%), ESR 92 $\mathrm{mm} / \mathrm{s}(0-20)$, procalcitonin $0.8776 \mathrm{ng} / \mathrm{ml}(<0.1)$, total protein $5.66 \mathrm{~g} / \mathrm{dl}(6.4-8.3)$, albumin $2.9 \mathrm{~g} / \mathrm{dl}$ (3.5-5), INR 1.15 (1-1.5), ferritin $2889 \mathrm{ng} / \mathrm{mL}$

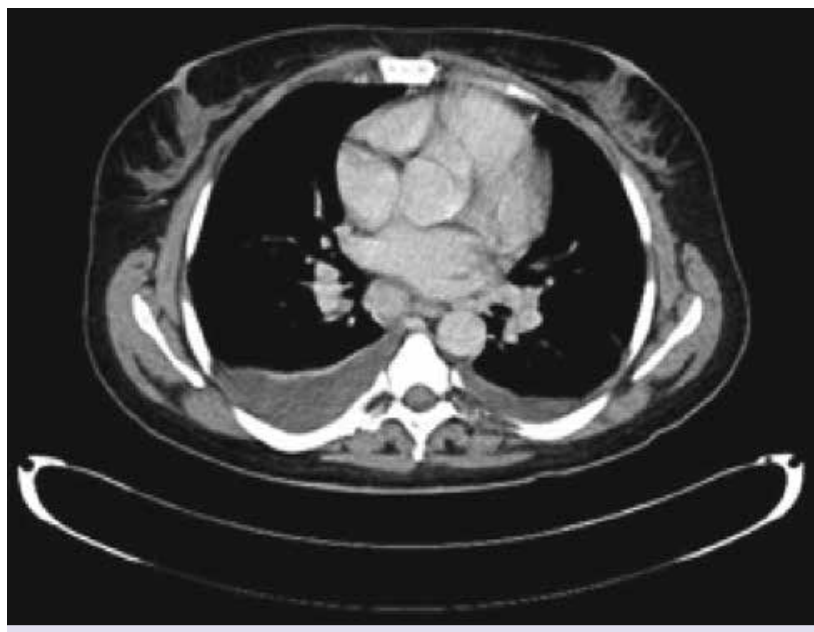

FIGURE 1. Bilateral pleural effusion on pre-treatment pulmonary tomogram.

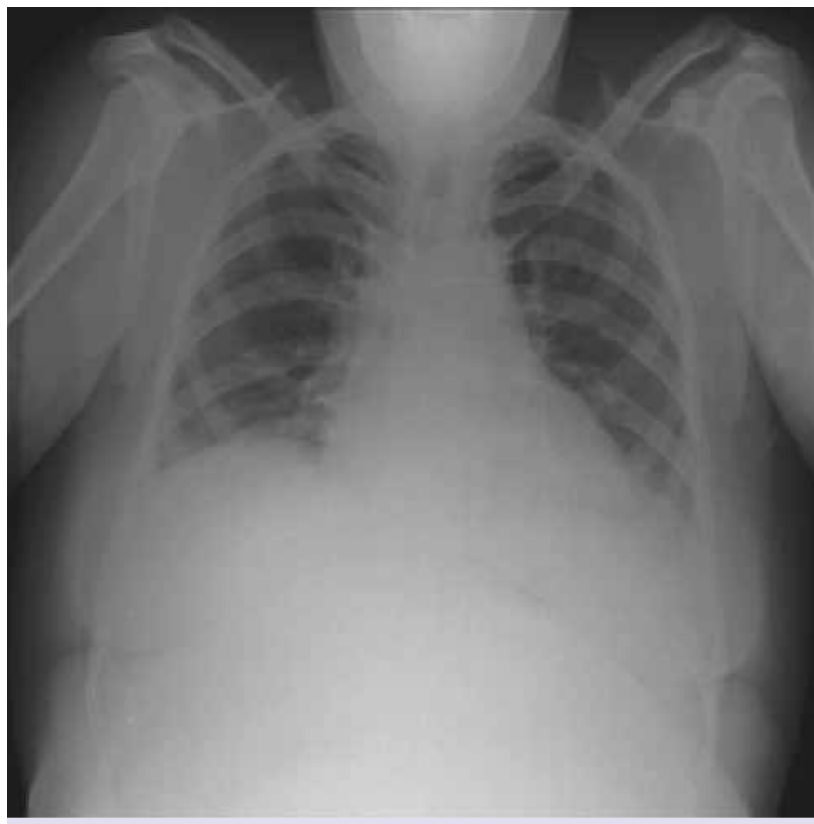

FIGURE2. On pre-treatment posteroanterior chest radiogram costodiaphragmatic sinuses are not distinctly visualized, high-lying diaphragms, and an enlarged mediastinum were observed.

(14-150). Glucose, creatinine, AST, ALT, ALP, Na, $\mathrm{K}$, complete urinalysis, ANA, RF, and ENA profile were unremarkable. On thoracal imagings, bilateral hilar lymph nodes of which the largest being $2 \mathrm{~cm}$ in diameter, bilateral pleural effusion, and atelectasic areas in the parenchyma surrounding the pleural effusion were observed (Figure 1,2). On abdomi- 
nal tomograms, only a few paraaortic, and paracaval lymph nodes were detected. No bacterial growth was detected in urine, throat, CSF, and blood cultures. Grubel Widal, and Brucella tube agglutination tests were unremarkable. Lumbar punction performed because of suspect neck rigidity did not reveal any abnormal cells in CSF. Febrile state of the patient persisted which necessitated initiation of an antibiotherapy (ie. cephtriaxone at daily parenteral doses of $2 \mathrm{~g}$ ). During follow-up period, rashes, and swelling on her right, and left wrists were detected, so she was consulted to rheumatology clinic, and naproxen sodium therapy was started with the indication of suspect AOSD. On physical examination dullness over Traube's space was noticed, and persistence of high fever required switch to imipenem therapy. However her hyperfebrile state persisted, echocardiograms obtained for the differential diagnosis of infective endocarditis.

Biochemical analysis of the fluid drained by thoracenthesis of the patient whose computed tomograms demonstrated bilateral pleural effusion revealed the presence of $2 \mathrm{gm} / \mathrm{dL}$ albumin, $3.34 \mathrm{~g} / \mathrm{dl}$ protein, 483 $\mathrm{u} / \mathrm{L} \mathrm{LDH}$ (concurrent serum albumin $2.9 \mathrm{gm} / \mathrm{dl}$, total protein $5.99 \mathrm{~g} / \mathrm{dl}$, LDH $393 \mathrm{u} / \mathrm{L}$ ). Antibiotherapy of the patient with pleural effusion (exudate) was discontinued, and methylprednisolone treatment at daily doses of $16 \mathrm{mg}$ was initiated.After initiation of steroid therapy, she maintained an afebrile state, her joint manifestations regressed, and her complaints decreased during her follow-up period. The patient whose pleural effusion completely regressed with steroid therapy discharged to be further followed up by rheumatology outpatient clinic (Figure 3).

\section{DISCUSSION}

Adult onset Still's disease is a disease whose diagnosis can be made by excluding infectious, malign, autoimmune, and autoinflammatory diseases, and in consideration of its characteristic clinical, and laboratory findings. For definitive diagnosis, Yamaguchi criteria can be used. Its major criteria consists of higher body temperature at $39^{\circ} \mathrm{C}$ lasting for at least one week, marked nonpruritic macular/maculopapular salmon-colored rashes on the torso or extremities persisting for at least 2 weeks, leukocytosis $(>10.000 / \mathrm{ml})$, and neutrophilia $(>80 \%)$. Minor criteria include sore throat, lymphadenopathy, hepato-

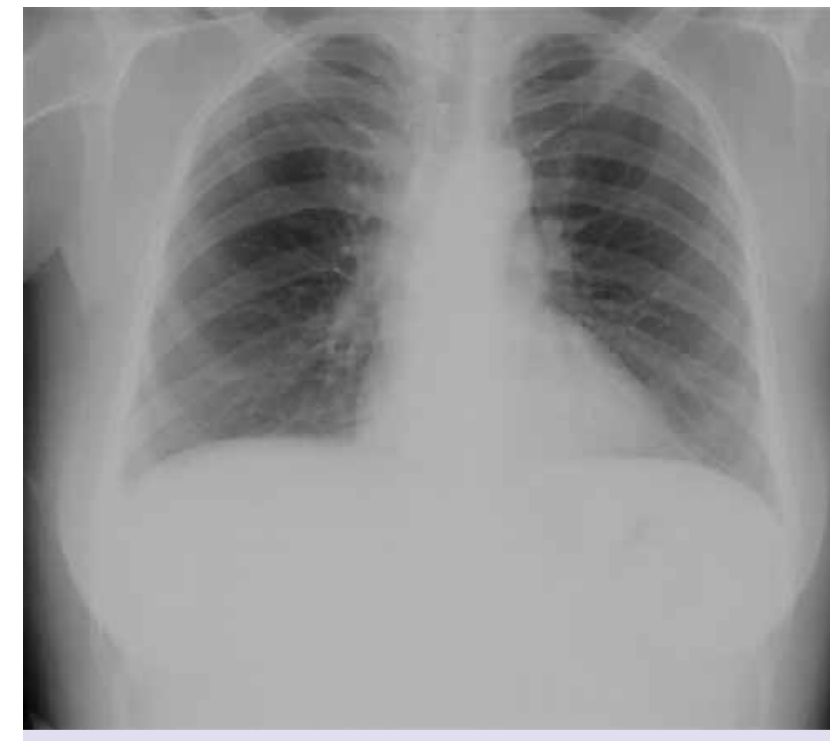

FIGURE 3. Post-treatment posteroanterior chest radiogram.

megaly or splenomegaly, abnormal hepatic function tests (increases in especially AST, ALT, and LDH levels), and ANA, and RF negativities. For the establishment of diagnosis, 5 diagnostic criteria (including at least 2 major criteria) should be met [5]. In our patient, during approximately 4 weeks up to initiation of steroid therapy, fever exceeding $39^{\circ} \mathrm{C} \mathrm{ev}$ ery day, symptoms of arthralgia, marked arthritis of both wrists, leukocytosis, neutrophilia, sore throat, and lymphadenopathies fulfilled the required diagnostic criteria, and pleural exudate seen during the clinical course of the disease was assessed to be associated with the disease.

In the adult onset Still's disease pleural effusion, transient pulmonary infiltration, and pericarditis can be seen in $30-40 \%$ of the patients. Affected individuals may complain of mild episodes of coughing, pleuritic chest pain, and shortness of breath. Rarely severe interstitial pulmonary disease can be seen. Even in some patients it may progress to Acute Respiratory Distress Syndrome (ARDS) [6, 7]. Rarely, myocarditis, arrhytmias, heart failure, and cardiac tamponade can be seen. Myocarditis, and ARDS are more frequently seen especially in patients with MAS.

In the differential diagnosis, infectious diseases (EBV, CMV, HBV, Rubella, Parvovirus, Coxsackie, HIV, Subacute Bacterial Endocarditis, Meningococ- 
cemia, Tuberculosis, Syphilis); vasculites ('Takayasu Arteritis, Polyarteritis Nodosa, Serum Disease, Thrombotic Thrombocytopenic Purpura); malign diseases (Leukemia, Lymphoma), connective tissue diseases (RA, SLE, Mixed Connective tissue Disease), granulomatous diseases (Sarcoidosis, Crohn disease); autoinflammatory diseases (Kikuchi disease, Sweet Syndrome), and drug hypersensitivity reactions should be considered. In our patient serological tests, cultures, and imaging modalities were used for differential diagnosis which led to the establishment of the diagnosis of AOSD.

Higher ferritin levels are among the most important laboratory findings of AOSD, however markedly lower glycosylated ferritin levels are detected $(<20 \%)$. Increased ferritin level is an indicator of the disease activity, and also a serologic marker of treatment response [8]. In our patient ferritin levels were measured twice, however glycosylated ferritin could not analyzed. Ferritin levels may increase as a response of hepatocytes to cytokines as IL-18, and IL-1B which may be thought to play a role in the pathogenesis of AOSD.

As the first-line therapy NSAIDs (especially endomethacin), and corticosteroids are recommended. In cases of severe organ involvement pulsatile high doses of steroids for few days can be used, than daily dose is dropped down to $1 \mathrm{mg} / \mathrm{kg}$. In cases of recurrence or with the intention to decrease steroid dose, methotrexate at a weekly dose of 7.5-25 mg can be given. Hydroxychloroquine, sulphasalazine, azothioprine, cyclosporine can be used separately or in combination with methotrexate. In recent years, effectiveness of biological agents (infliximab, etanercept, anakinra, and tocilizumab), and IVIG therapies have been reported in the treatment patients with serious organ involvement, and MAS [9].

Still many characteristic features of AOSD are not known, and a standard follow-up or a treatment protocol of the disease is not available. In patients who met diagnostic criteria, pleural effusion which may be seen in the clinical course of the disease should be presumably associated with the disease. Besides regression of pleural effusion can be expected with the treatment of underlying desease, additional locally invasive diagnostic, and/or therapeutic interventions should be avoided.

Conflict of Interest: No conflict of interest was declared by the authors.

Financial Disclosure: The authors declared that this study has received no financial support.

\section{REFERENCES}

1. Efthimiou P, Paik PK, Bielory L. Diagnosis and management of adult onset Still's disease. Ann Rheum Dis 2006;65:564-72.

2. Altıntop L, Kut E, Çilingir FM, Yıldırım A, Gökdemir R. A notable reason for fever of unknown origin: Adult onset still disease. Journal of Clinical and Experimental Investigations 2013;4:119-22.

3. Gerfaud-Valentin M, Maucort-Boulch D, Hot A, Iwaz J, Ninet J, Durieu I, et al. Adult-onset still disease: manifestations, treatment, outcome, and prognostic factors in 57 patients. Medicine (Baltimore) 2014;93:91-9.

4. Hot A, Toh ML, Coppéré B, Perard L, Madoux MH, Mausservey $\mathrm{C}$, et al. Reactive he-mophagocytic syndrome in adult-onset Still disease: clinical features and long-term outcome: a case-control study of 8 patients. Medicine (Baltimore) 2010;89:37-46.

5. Yamaguchi M, Ohta A, Tsunematsu T, Kasukawa R, Mizushima Y, Kashiwagi $\mathrm{H}$, et al. Preliminary criteria for classification of adult Still's disease. J Rheumatol 1992;19:424-30.

6. Cheema GS, Quismorio FP Jr. Pulmonary involvement in adultonset Still's disease. Curr Opin Pulm Med 1999;5:305-9.

7. Manganelli P, Fietta P, Zuccoli P. Adult-onset Still's disease with respiratory distress syndrome, polyserositis and disseminated intravascular coagulation: a case with a fatal outcome. Clin Exp Rheumatol 2003;21:139.

8. Kádár J, Petrovicz E. Adult-onset Still's disease. Best Pract Res Clin Rheumatol 2004;18:663-76.

9. Gerfaud-Valentin M, Jamilloux Y, Iwaz J, Sève P. Adult-onset Still's disease. Autoimmun Rev 2014;13:708-22. 